\title{
Insights into the Solubility of Poly(vinylphenothiazine) in Carbonate- Based Battery Electrolytes
}

Verena Perner ${ }^{a}$, Diddo Diddens ${ }^{b}$, Fabian Otteny ${ }^{c}$, Verena Küpers ${ }^{a}$, Peter Bieker ${ }^{a, b}$, Birgit Esser $^{c, d, ~ e}$, Martin Winter ${ }^{\mathrm{a}, \mathrm{b}}$ and Martin Kolek ${ }^{\mathrm{a}}$

a MEET Battery Research Center, Institute of Physical Chemistry, University of Münster, Corrensstraße 46, 48149 Münster, Germany

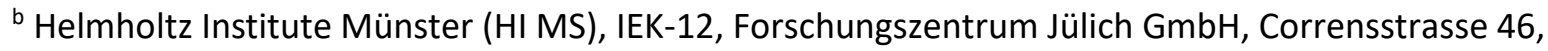
48149 Münster, Germany

${ }^{c}$ Institute for Organic Chemistry, University of Freiburg, Albertstraße 21, 79104 Freiburg, Germany

${ }^{d}$ Freiburg Materials Research Center, University of Freiburg, Stefan-Meier-Straße 19, 79104 Freiburg, Germany

e Freiburg Center for Interactive Materials and Bioinspired Technologies, University of Freiburg, Georges-Köhler-Allee 105, 79110 Freiburg, Germany

*Correspondence: martin.kolek@uni-muenster.de, besser@oc.uni-freiburg.de 


\section{Table of Contents}

1 Constant Current Cycling Investigations on PVMPT-Based Composite Electrodes - Variation of

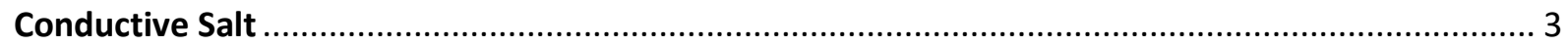

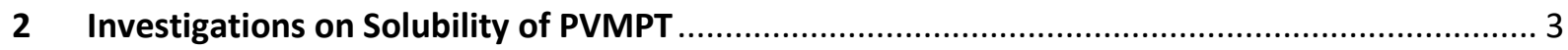

3 Constant Current Cycling Investigations on PVMPT-Based Composite Electrodes - $1 \mathrm{M} \mathrm{LiPF}_{6}$ in

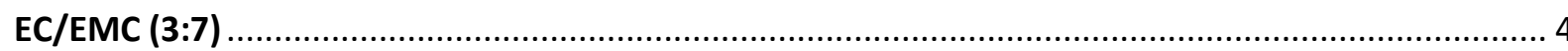

4 Rate Capability Investigations on PVMPT-Based Composite Electrodes - $1 \mathrm{M} \mathrm{LiPF}_{6}$ in EC/DMC (1:1) and EC/EMC (3:7)

5 Investigations on Self-Discharge of PVMPT-Based Composite Electrodes - $1 \mathrm{M} \mathrm{LiPF}_{6}$ in

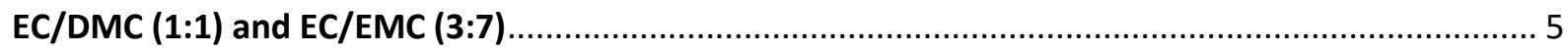

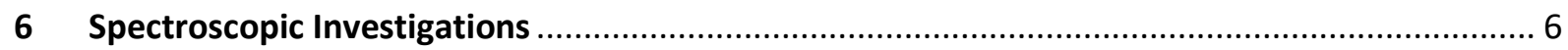

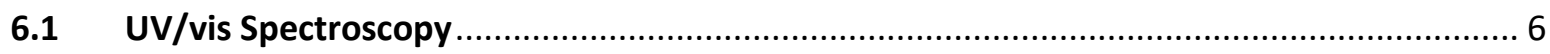

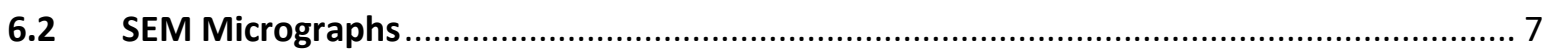

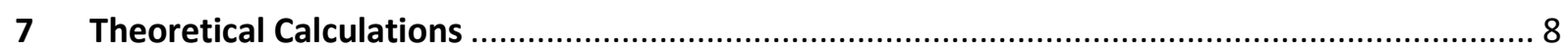

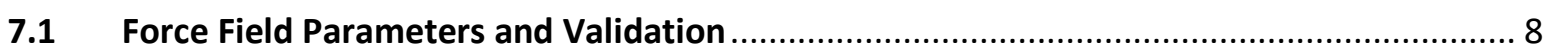

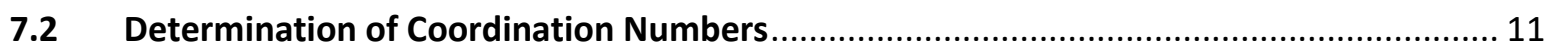

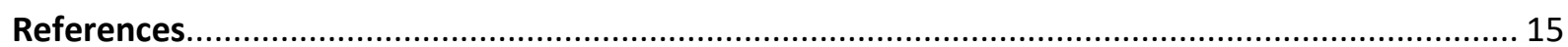




\section{Constant Current Cycling Investigations on PVMPT-Based Composite Electrodes - Variation of Conductive Salt}

All composite electrodes were fabricated as described in the Experimental Procedure section of the manuscript.

(a)

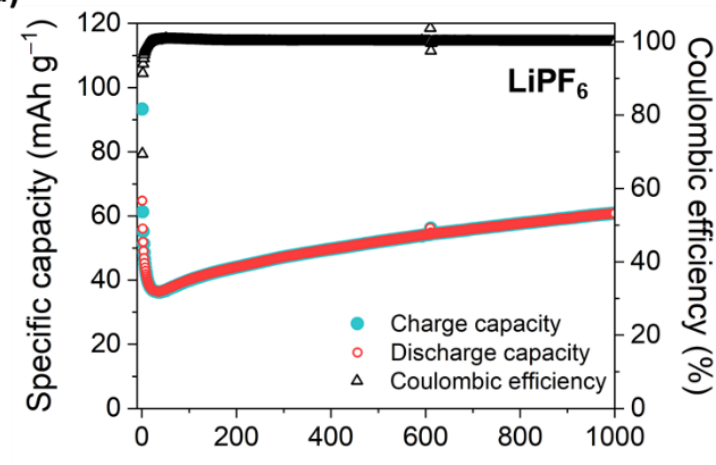

(c)

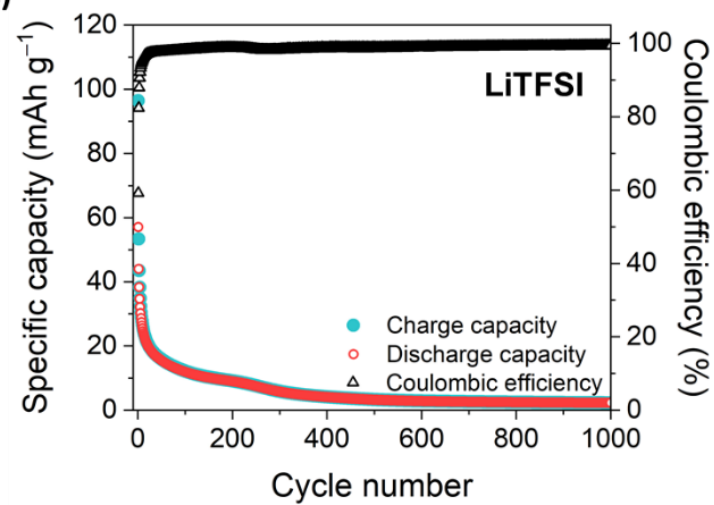

(b)

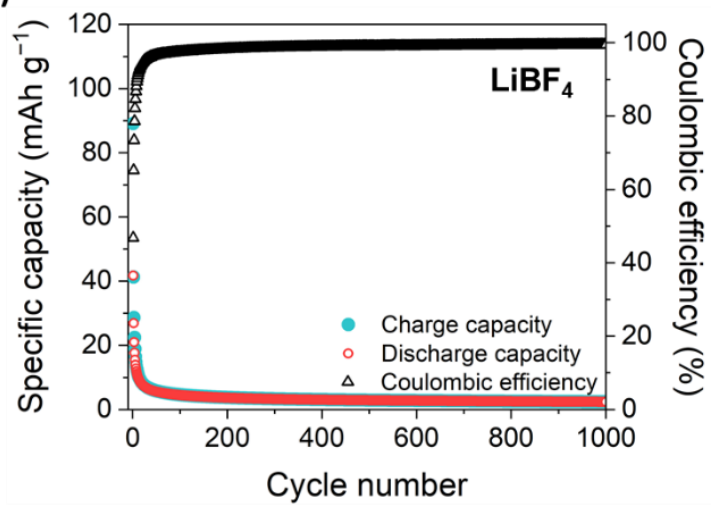

Figure S1. Specific capacity and Coulombic efficiency vs cycle number plots of constant current cycling investigations of a PVMPT-based electrode using different salts (a) LiPF 6 , (b) LiBF 4 and (c) LiTFSI in EC/DMC (1:1) at $1 \mathrm{C}$ rate (1 $\mathrm{M}$ each).

\section{Investigations on Solubility of PVMPT}

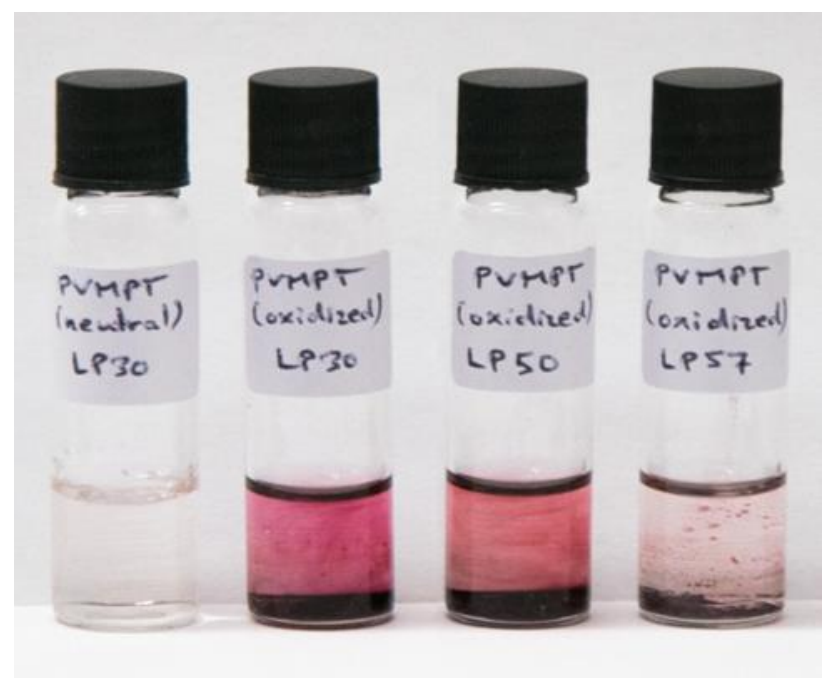

Figure S2. Solubility test of PVMPT in the battery electrolytes EC/DMC 1:1 (LP30), EC/EMC 1:1 (LP50), and EC/EMC 3:7 (LP57) with $1 \mathrm{M} \mathrm{LiPF} 6$ each in the neutral (for EC/DMC 1:1) and oxidized state. 


\section{Constant Current Cycling Investigations on PVMPT-Based Composite Electrodes - $1 \mathrm{M} \mathrm{LiPF}_{6}$ in EC/EMC (3:7)}

All composite electrodes were fabricated as described in the Experimental Procedure section of the manuscript.

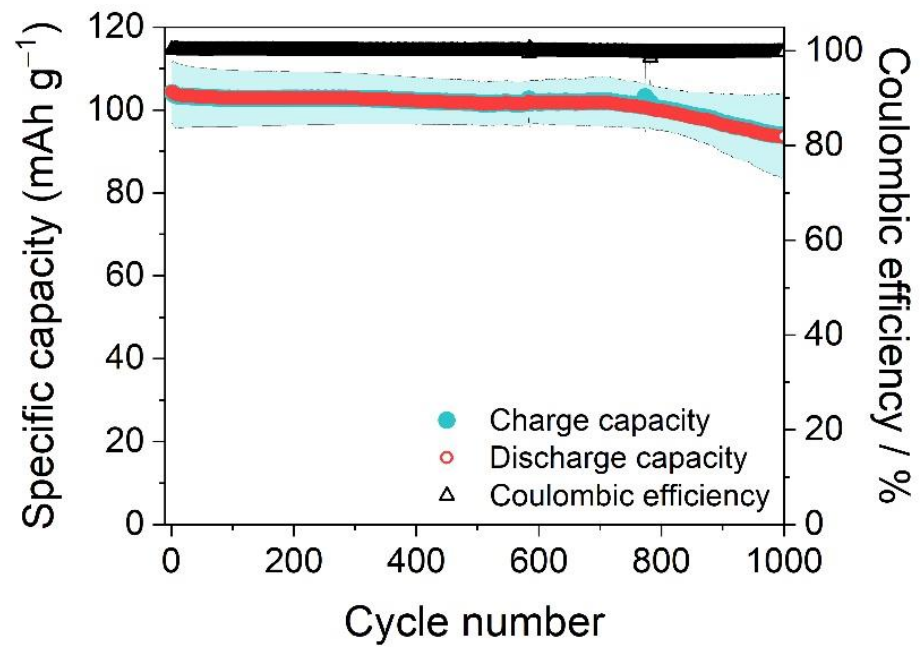

Figure S3. Specific capacity and Coulombic efficiency vs cycle number plot of constant current cycling investigations of PVMPT-based electrodes using the solvent mixture EC/EMC (3:7) with $1 \mathrm{M}$ LiPF $_{6}$ at a $1 \mathrm{C}$ rate, including standard deviation over three measurements.

\section{Rate Capability Investigations on PVMPT-Based Composite Electrodes - $1 \mathrm{M} \mathrm{LiPF}_{6}$ in EC/DMC (1:1) and EC/EMC (3:7)}

(a)

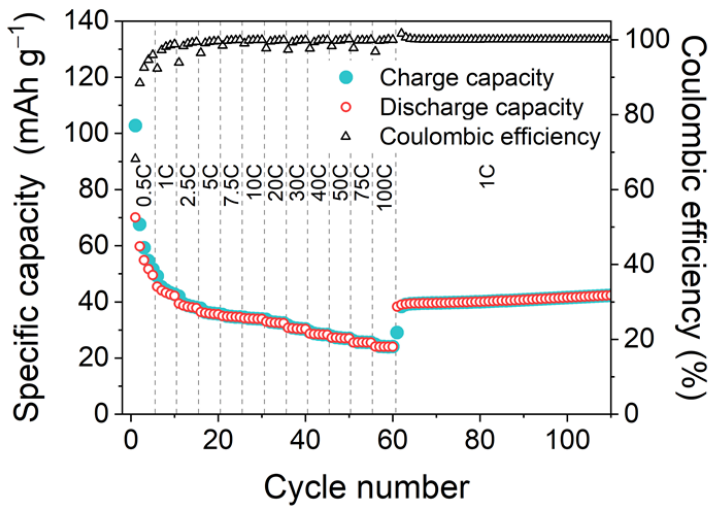

(b)

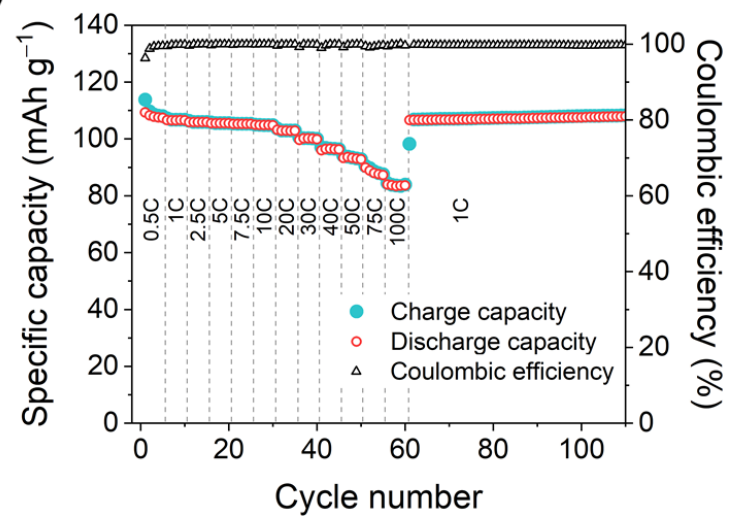

Figure S4. Specific capacity and Coulombic efficiency vs cycle number plots of investigations on rate capability of PVMPT-based electrodes using the solvent mixture (a) EC/DMC (1:1) and (b) EC/EMC (3:7) with 1 M LiPF6. 


\section{Investigations on Self-Discharge of PVMPT-Based Composite Electrodes - $1 \mathrm{M} \mathrm{LiPF}_{6}$ in EC/DMC (1:1) and EC/EMC (3:7)}

(a)

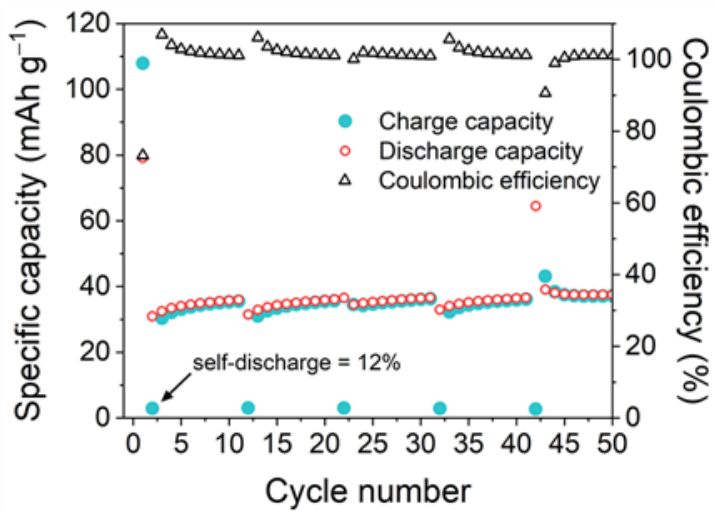

(b)

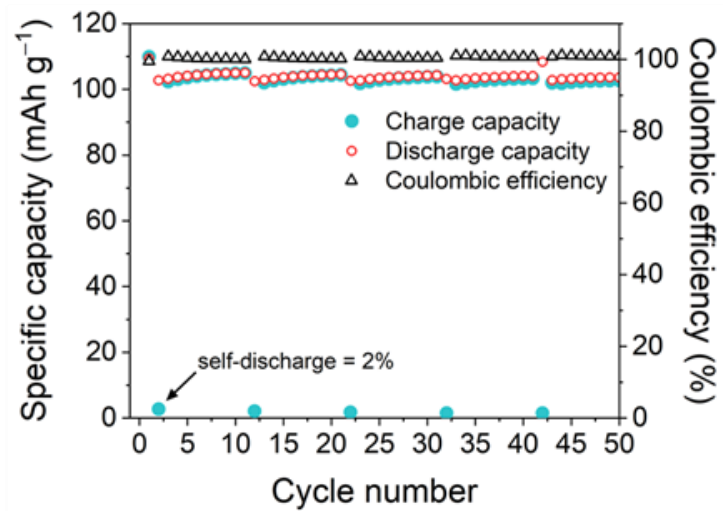

Figure S5. Specific capacity and Coulombic efficiency vs cycle number plots of investigations on self-discharge of PVMPT-based electrodes using the solvent mixture (a) EC/DMC (1:1) and (b) EC/EMC (3:7) with $1 \mathrm{M} \mathrm{LiPF.}$

PVMPT-based electrodes (50 wt\%) were cycled in a potential range of $3.1-3.9 \mathrm{~V}$ vs $\mathrm{Li}_{\mid} \mathrm{Li}^{+}$at a $1 \mathrm{C}$ rate. After full charge, the cell was rested for $24 \mathrm{~h}$ under open circuit potential conditions. Hereafter, the cell was fully charged under the same conditions and the lost charge capacity was recorded. The cell was cycled further for 10 cycles, and the procedure was repeated. In order to obtain the amount of self-discharge, the specific capacities after the resting steps were divided by the charge capacity before the resting step. 


\section{Spectroscopic Investigations}

\subsection{UV/vis Spectroscopy}

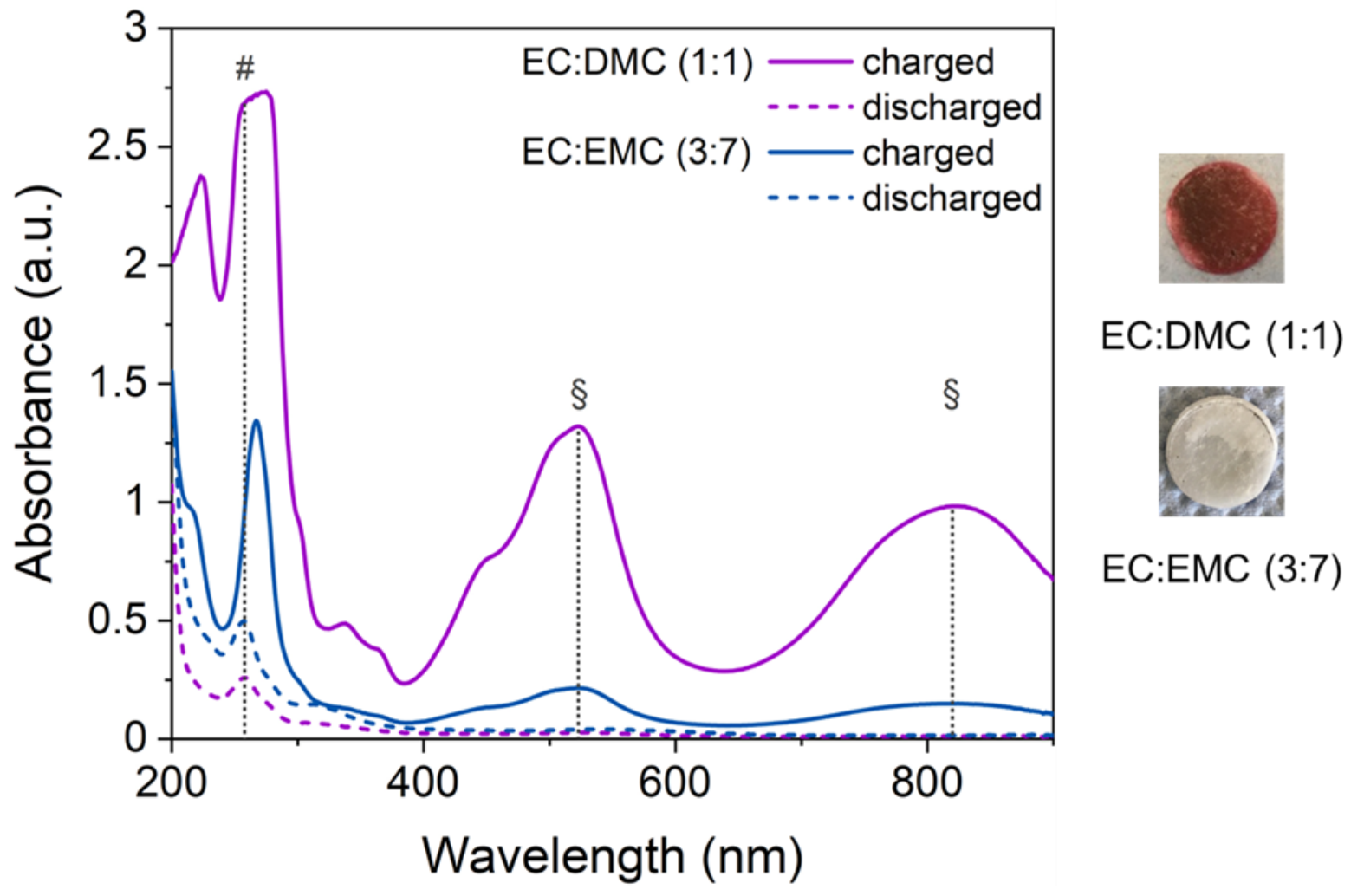

Figure S6. UV/vis spectra of electrolytes extracted from initially charged or discharged PVMPT-based battery cells; \# indicates bands for neutral PVMPT, $\S$ indicates bands for oxidized PVMPT (radical cation of MPT at $520 \mathrm{~nm}$, dimer of PVMPT (oxidation state C) at $820 \mathrm{~nm}$ ). Right side: Photographs of separators obtained from respective, disassembled battery cells in the charged state. 


\subsection{SEM Micrographs}

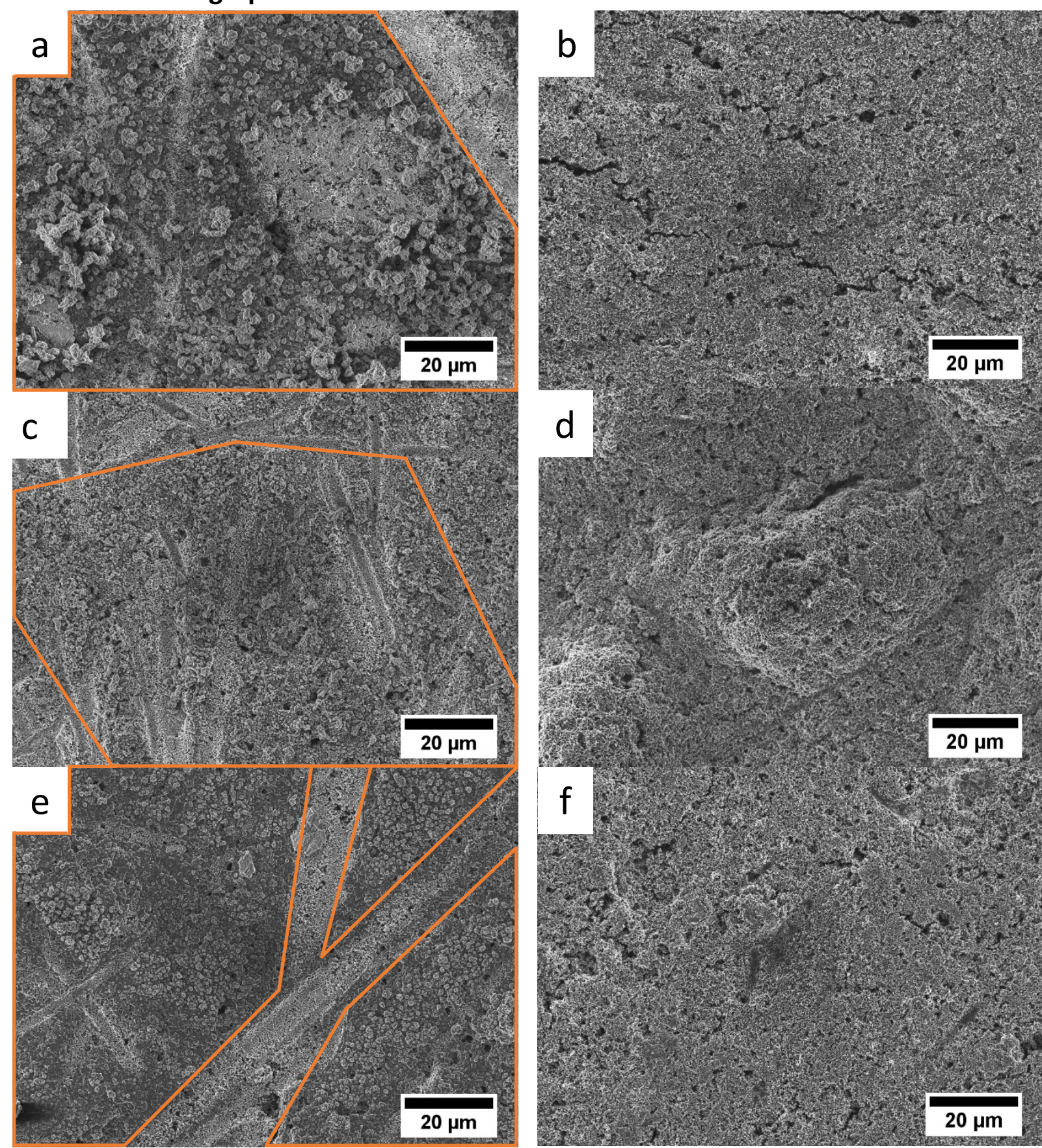

Figure S7. SEM micrographs of PVMPT-based electrodes using EC/EMC (1:1) (a, c, e) or EC/EMC (3:7) (b, d, f) as electrolyte solvents: after 1 cycle $(a, b), 50$ cycles (c, d), and 100 cycles $(e, f)$ in the discharged state. The deposits of PVMPT were highlighted by orange markers (compare Figure 6 ). 


\section{Theoretical Calculations}

\subsection{Force Field Parameters and Validation}

Figure $\mathrm{S} 8$ shows the partial charges for EC, EMC, DMC, as well as a neutral and oxidized PVMPT, as determined by the ChelpG method at the MP2/cc-pVTZ level of theory on molecule geometries optimized at the PBEO-D3BJ/def2-TZVP level. ${ }^{1-6}$ The calculations were performed with Gaussian $16 .^{7}$ We note that for the carbonates, our charges are similar to those derived by Borges Silva et al.. ${ }^{8}$

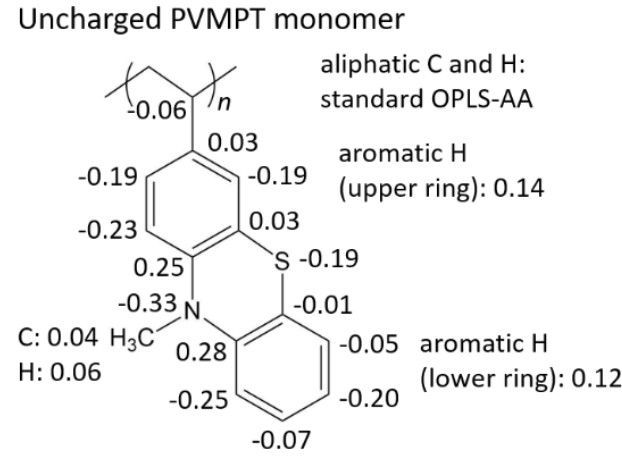

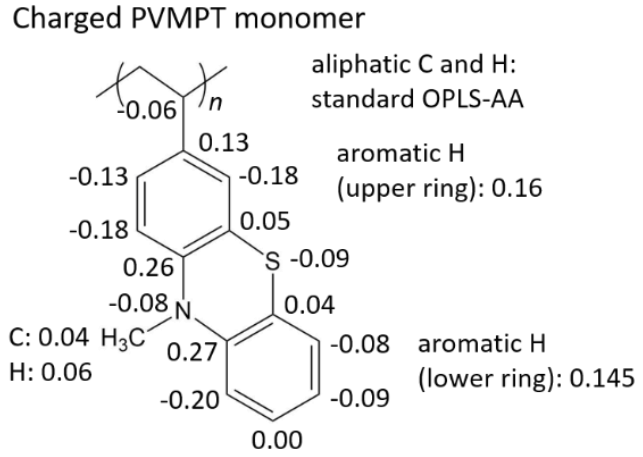

Ethylene carbonate

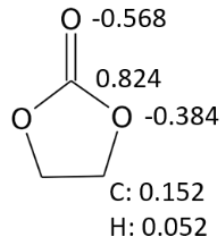

Ethyl methyl carbonate

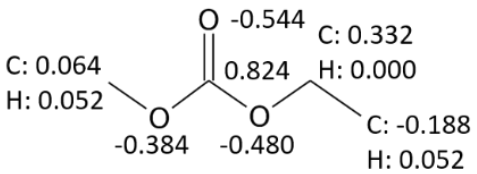

$\mathrm{H}: 0.052$
Dimethyl carbonate

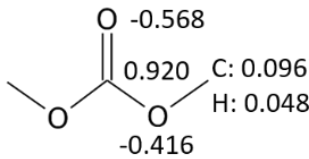

Figure S8. Partial charges for charged and uncharged PVMPT monomers, as well as the carbonates EC, EMC and DMC employed in this study. The partial charges of the PVMPT backbone atoms correspond to the standard OPLS-AA charges, whereas the partial charges for $\mathrm{PF}_{6}{ }^{-}$were taken from the CL\&P force field..$^{9-12}$

The Lennard-Jones (L) parameters and bonded interactions for PVMPT, EC, EMC, and DMC were taken from the OPLS-AA force field, and from the CL\&P force field for $\mathrm{LiPF}_{6}$ (the $\mathrm{L}$ parameters for $\mathrm{Li}^{+}$ corresponding to those of Åqvist). ${ }^{9-13}$

The structure of the central ring of the PVMPT monomers was stabilized by adding a virtual bond between the nitrogen and the sulfur atom, that was constrained to a length of $0.309 \mathrm{~nm}$ (charged form) or $0.297 \mathrm{~nm}$ (neutral form). Furthermore, the dihedrals formed by this virtual bond and the adjacent carbon atoms of the other two aromatic rings were restrained by a harmonic potential (force constant $60.668 \mathrm{~kJ} \mathrm{~mol}^{-1} \mathrm{rad}^{-2}$ ) to equilibrium values of $\pm 0.4^{\circ}$ and $\pm 164.3^{\circ}$ (charged form) or $\pm 1.7^{\circ}$ and $\pm 138.8^{\circ}$ (neutral form). Dihedrals between the carbon atom of the methyl group attached to nitrogen atom and other aromatic carbon atoms were set to $\pm 14.9^{\circ}$ and $\pm 164.7^{\circ}$ (charged form) or $\pm 13.4^{\circ}$ and $\pm 166.2^{\circ}$ (neutral form). The improper dihedral defined by the nitrogen atom and all three connected carbon atoms was harmonically restrained to values of $\pm 1.3^{\circ}$ and $\pm 14.9^{\circ}$ (neutral form). Finally, to mimic $\pi$ interactions between charged PVMPT monomers, the respective sulfur and nitrogen atoms of adjacent monomers were connected by harmonic springs with equilibriums lengths of $0.370 \mathrm{~nm}$ 
(nitrogen-nitrogen bond) and $0.318 \mathrm{~nm}$ (sulfur-sulfur bond) and a harmonic force constant of $10,000 \mathrm{~kJ} \mathrm{~mol}^{-1} \mathrm{~nm}^{-2}$. All above-mentioned distances and dihedral angles were derived from the PBE0-D3BJ/def2-TZVP geometries of charged and uncharged PVMPT monomers or charged dimers (modeling of $\pi$-interactions). All other dihedrals and improper dihedrals of PVMPT were taken from the original OPLS-AA force field.

Additionally, missing dihedral parameters for the carbonates were derived from relaxed potential energy scans at the PBEO-D3BJ/def2-TZVP level followed by single-point calculations at the MP2/cc-pVTZ level of theory. ${ }^{4-6}$ Subsequently, the structures of the scan were minimized in short Molecular Dynamics (MD) runs, in which the corresponding dihedrals were restrained and their force constant set to zero. ${ }^{14}$ The difference in energy between MP2 calculations and MD simulations was then fitted to the function

$$
V_{d i h}=\frac{1}{2}\left[C_{1}(1+\cos (\phi))+C_{2}(1-\cos (2 \phi))+C_{3}(1+\cos (3 \phi))+C_{4}(1-\cos (4 \phi))\right]
$$

to obtain the force field parameters. ${ }^{10-12}$ The results are shown in Figure S9, the fit parameters are listed in Table S1. Note that the force constants of the $\mathrm{C}-\mathrm{O}-\mathrm{C}_{\mathrm{carb}}-\mathrm{O}$ dihedral (where the oxygens correspond to the ether-like oxygens of the carbonate group) was set to zero, and all energy contributions were adsorbed in the $\mathrm{C}-\mathrm{O}-\mathrm{C}_{\mathrm{carb}}-\mathrm{O}_{\text {carb }}$ dihedral.

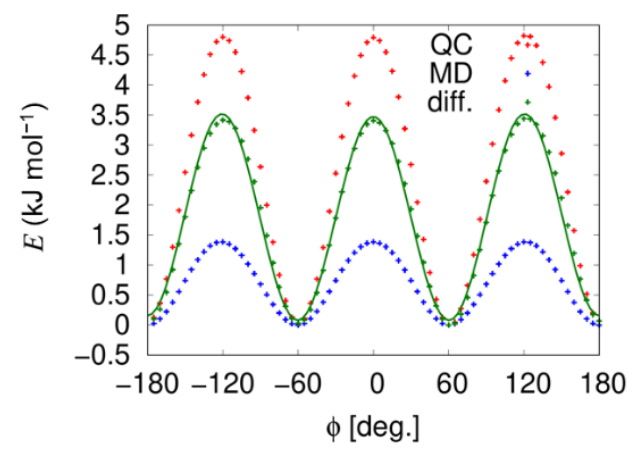

DMC: H-C-O-C $\mathrm{Carb}_{\text {dihedral }}$

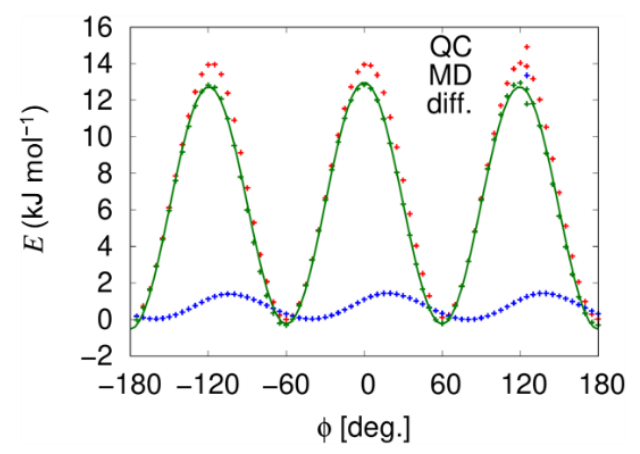

EMC: H-C-C-O dihedral

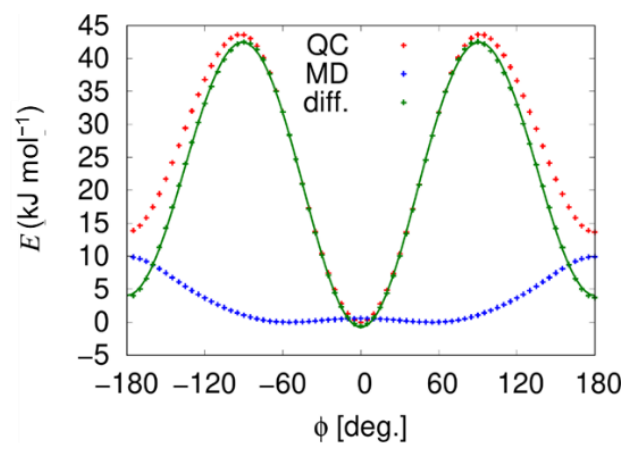

DMC: C-O- $\mathrm{C}_{\text {carb }}-\mathrm{O}_{\text {carb }}$ dihedral

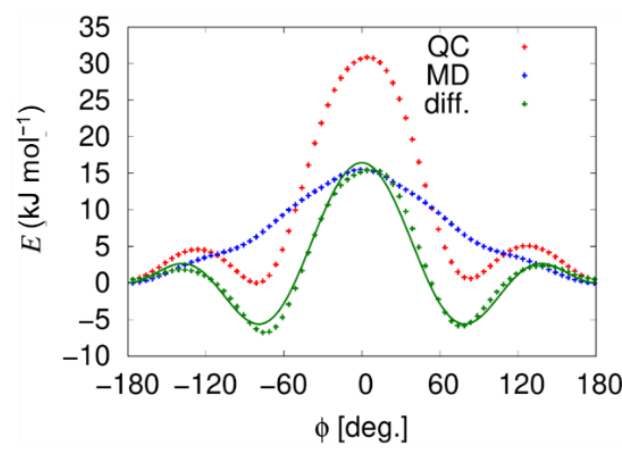

EMC: C-C-O-C carb dihedral

Figure 59. Potential energy profiles for the rotation around various bonds of DMC and EMC. The quantum chemical results were obtained from relaxed scans at the PBE0-D3BJ/def2-TZVP level of theory, followed by single-point calculations at the MP2/cc-pVTZ level. The solid lines correspond to fits of the differences between quantum chemical and MD curves. 
Table S1. Dihedral fit parameters from Figure S9. Note that the H-C-C-O dihedral for EMC is equivalent to the respective OPLS-AA dihedral for ethers.

$\begin{array}{lcccc}\text { Dihedral } & C_{1}\left(\mathrm{~kJ} \mathrm{~mol}^{-1}\right) & C_{2}\left(\mathrm{~kJ} \mathrm{~mol}^{-1}\right) & C_{3}\left(\mathrm{~kJ} \mathrm{~mol}^{-1}\right) & C_{4}\left(\mathrm{~kJ} \mathrm{~mol}^{-1}\right) \\ \text { DMC: H-C-O-C carb } & 0.00 & 0.00 & 0.57 & 0.00 \\ \text { DMC: C-O-C carb-O } & -2.11 & 20.40 & -0.30 & 0.18 \\ \text { EMC: H-C-C-O } & 0.00 & 0.00 & 1.96 & 0.00 \\ \text { EMC: C-C-O-C carb } & 4.16 & -6.55 & 4.05 & 0.49\end{array}$

To validate our simulation parameters, we additionally simulated two EC/EMC mixtures with weight ratios of 1:1 and 3:7, respectively, consisting of $400 \mathrm{EC}$ and $340 \mathrm{EMC}$ or $250 \mathrm{EC}$ and $500 \mathrm{EMC}$ molecules. For these systems, we computed the relative permittivity or dielectric constant $\varepsilon_{\mathrm{r}}$ according to

$$
\varepsilon_{\mathrm{r}}=1+\frac{1}{3} \frac{\left\langle M^{2}\right\rangle}{V k_{\mathrm{B}} T}
$$

where $M$ is the total dipole moment in the simulation box with volume $V$, and $k_{\mathrm{B}} T$ the thermal energy. ${ }^{15,16}$

Table S2 compares the results to experimentally determined values. ${ }^{17}$ We note that the simulations slightly underestimate the experimentally measured $\varepsilon_{\mathrm{r}}$ values, however, we deem the accuracy sufficient for our purpose. Potential refinements of the simulation model could be introduced via the incorporation of electronic polarization. ${ }^{18}$

Table S2. Computed and experimentally determined values of the dielectric constant $\varepsilon_{\mathrm{r}}$ of EC/EMC blends with weight ratios of $1: 1$ and 3:7 at $\mathrm{T}=298 \mathrm{~K} .{ }^{17}$

\section{EC/EMC ratio \\ (by weight)}

1:1

$3: 7$

\section{Dielectric constant $\varepsilon_{\mathrm{r}}$ Experiment Simulation}

33.6

18.5
28.9

14.6

Furthermore, we performed simulations of the two EC/EMC blends and two analogous EC/DMC blends with $1 \mathrm{M} \mathrm{LiPF}_{6}$. For the EC/EMC ratio of 1:1, the system consisted of $400 \mathrm{EC}$ and $340 \mathrm{EMC}$ molecules with $70 \mathrm{LiPF}_{6}$ ion pairs, while the system with a 3:7 ratio was composed of $250 \mathrm{EC}$ and $500 \mathrm{EMC}$ molecules with 75 LiPF $_{6}$ ion pairs. The EC/DMC (1:1) system consisted of 400 EC and 400 DMC molecules with $70 \mathrm{LiPF}_{6}$ ion pairs, and the EC/DMC (3:7) system of $240 \mathrm{EC}$ and $560 \mathrm{EMC}$ molecules with $70 \mathrm{LiPF}_{6}$ ion pairs. Based on the radial distribution functions (not shown), we computed the number of nearest neighbours around the lithium ions (Figure S10). We note that the resulting coordination numbers are in good agreement with previous simulation studies, demonstrating that our force field is sufficiently accurate to describe the solution environment properly. ${ }^{19-21}$ 
The simulations of a PVMPT chain immersed in EC/EMC or EC/DMC consisted of one PVMPT chain with 24 monomers, 1600 EC and 1360 EMC molecules for EC/EMC (1:1), 930 EC and 1870 EMC molecules for EC/EMC (3:7), $1600 \mathrm{EC}$ and $1600 \mathrm{DMC}$ molecules for EC/DMC (1:1), as well as $960 \mathrm{EC}$ and 2240 DMC molecules for EC/DMC (3:7). In all cases, $280 \mathrm{LiPF}_{6}$ ion pairs were added to obtain a $1 \mathrm{M}$ $\mathrm{LiPF}_{6}$ solution. The excess charge of PVMPT was compensated by additional $\mathrm{PF}_{6}{ }^{-}$counterions. The final simulation boxes had a length of about $7.6 \mathrm{~nm}$.

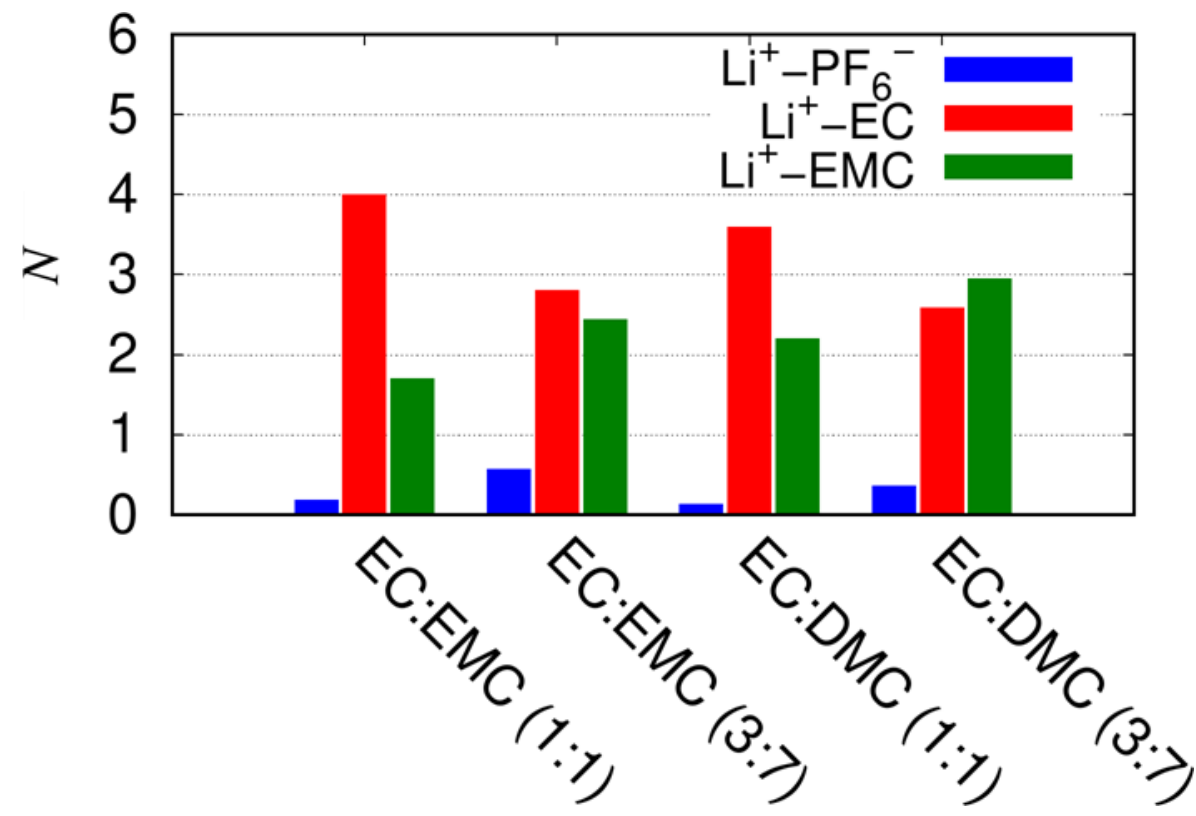

Figure S10. Lithium ion coordination numbers in various EC/EMC and EC/DMC electrolytes containing 1 м LiPF6.

\subsection{Determination of Coordination Numbers}

To compute the number of EC/EMC molecules and $\mathrm{PF}_{6}{ }^{-}$ions in the vicinity of the positively charged PVMPT chain, we calculated the radial distribution functions (RDFs) between the sulfur atoms of PVMPT and the carbon atoms of the carbonate group of EC and EMC as well as the phosphorus atom of $\mathrm{PF}_{6}{ }^{-}$(Figure S11). For EC and EMC, we observe a first coordination peak at $0.42-0.45 \mathrm{~nm}$ with a subsequent minimum at $0.64 \mathrm{~nm}$, while for $\mathrm{PF}_{6}{ }^{-}$a pronounced peak at $0.48 \mathrm{~nm}$ with a subsequent minimum at $0.72 \mathrm{~nm}$ is observed for $\mathrm{PF}_{6}{ }^{-}$, followed by a second peak at $0.83 \mathrm{~nm}$, arising from the strong coordination of multiple $\mathrm{PF}_{6}{ }^{-}$anions to a given monomer or its neighbors. For $\mathrm{EC}$ and EMC, a minor second peak can be observed at comparable distances. Remarkably, the positions of the peaks and the minima hardly change with the EC/EMC ratio or the length of the sequences stabilized by $\pi$ interactions. Therefore, we determined the coordination numbers from the volume integrals of the RDFs up to the same respective positions of the minima for both compositions and for all monomer sequences stabilized by $\pi$-interactions, which are shown in Figures $7 a-c$ in the main text. For the EC/DMC electrolytes (Figure S12), basically identical peak positions are observed, and the respective coordination numbers were determined in analogy to Figure S11. For neutral PVMPT (Figure S13), 
$\pi$-interactions are irrelevant and hence ignored in the simulations. ${ }^{22}$ We observe no significant coordination of either $\mathrm{EC}, \mathrm{EMC}$, or $\mathrm{PF}_{6}{ }^{-}$from the RDFs (Figures $\mathrm{S} 13 \mathrm{a}$ and $\mathrm{b}$ ). Nonetheless, to facilitate comparison with the coordination numbers of charged PVMPT, we adopted the same distance criteria as above for the coordination numbers in Figure S10.

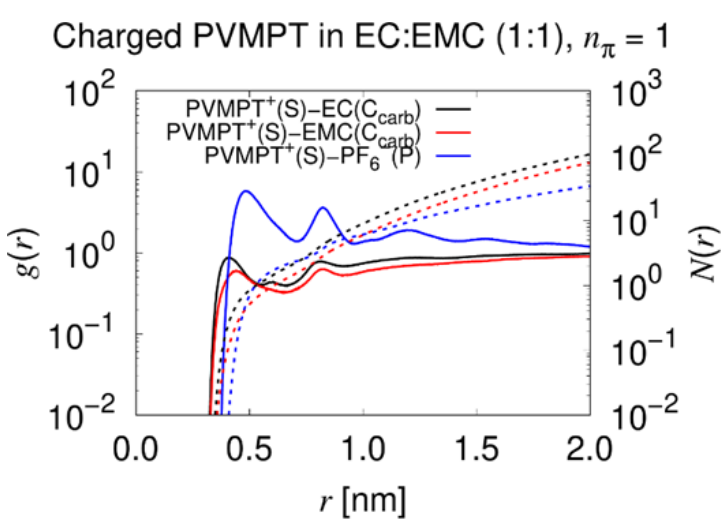

Charged PVMPT in EC:EMC (1:1), $n_{\pi}=2$

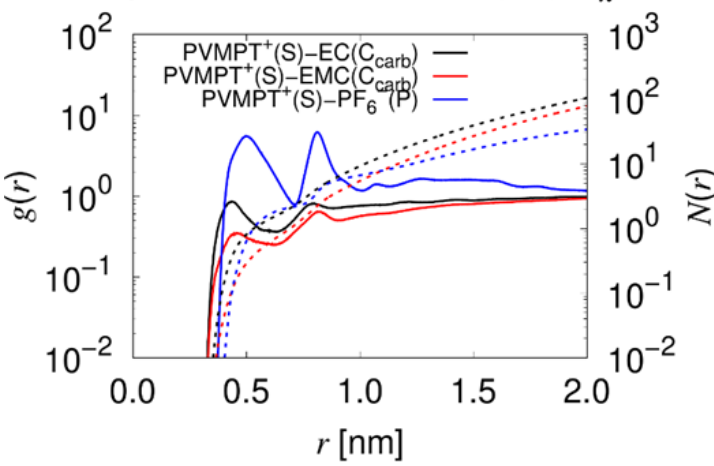

Charged PVMPT in EC:EMC (1:1), $n_{\pi}=3$

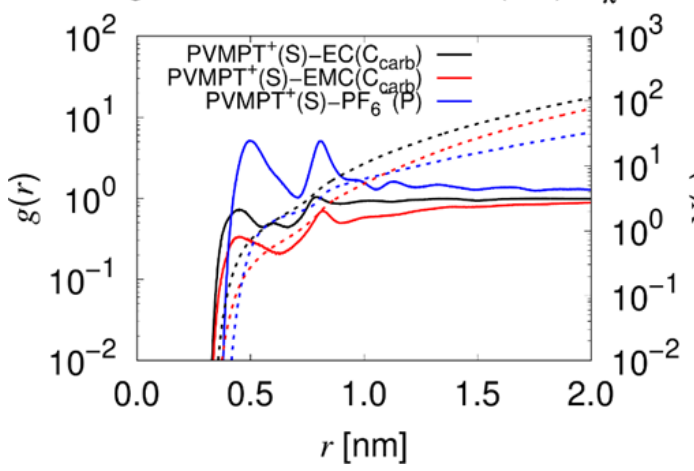

Charged PVMPT in EC:EMC (3:7), $n_{\pi}=1$

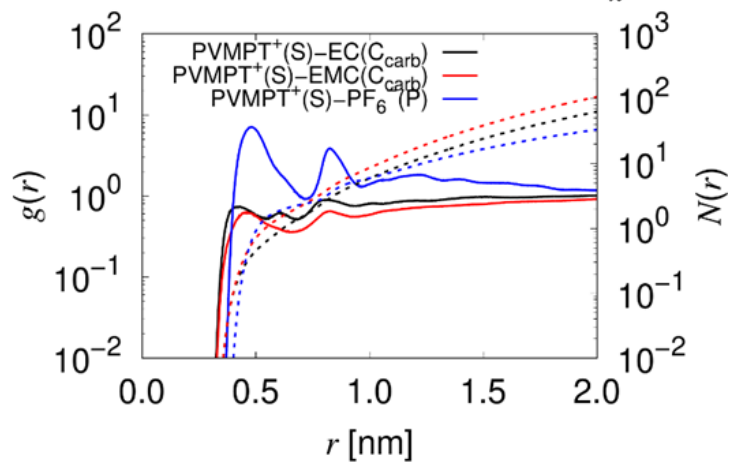

Charged PVMPT in EC:EMC (3:7), $n_{\pi}=2$

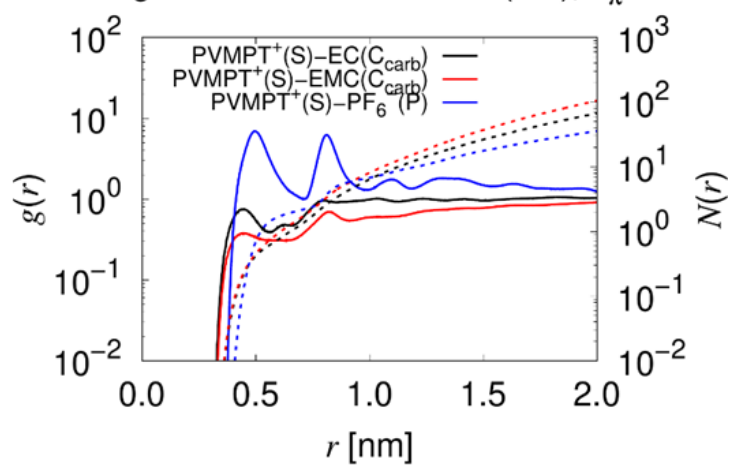

Charged PVMPT in EC:EMC (3:7), $n_{\pi}=3$

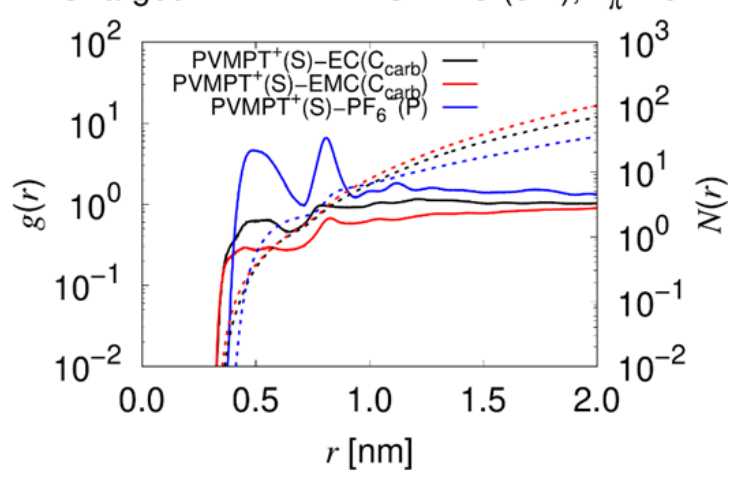

Figure S11. Radial distribution functions $g(r)$ (solid) and distance-dependent coordination numbers $N(r)$ (dashed) between the sulphur atoms of a fully oxidized PVMPT chain and the carbon atoms of the carbonate group of $E C$ and $E M C$ or the phosphorus atom of $\mathrm{PF}_{6}{ }^{-}$in $E C / E M C$ electrolytes. Both mixing ratios EC/EMC (1:1) and EC/EMC (3:7) as well as chains with monomer sequences of various length that are stabilized by $\pi$ interactions are compared. 


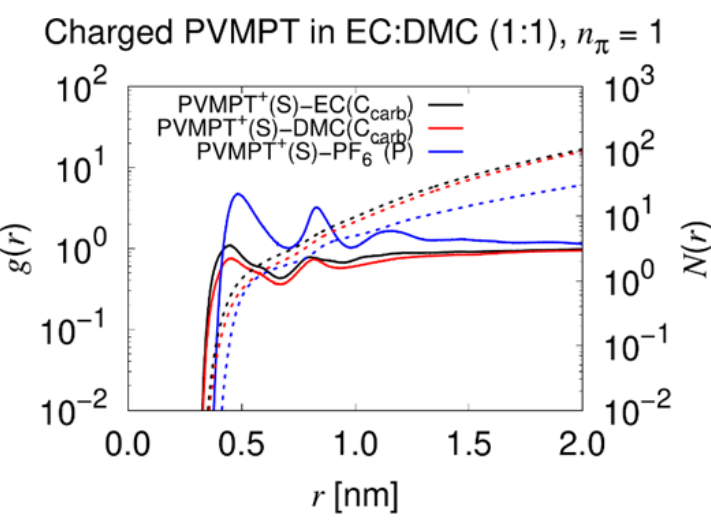

Charged PVMPT in EC:DMC $(1: 1), n_{\pi}=2$

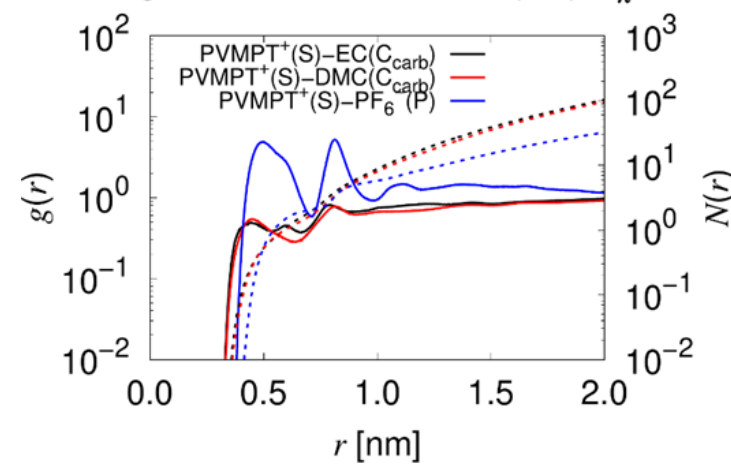

Charged PVMPT in EC:DMC (1:1), $n_{\pi}=3$

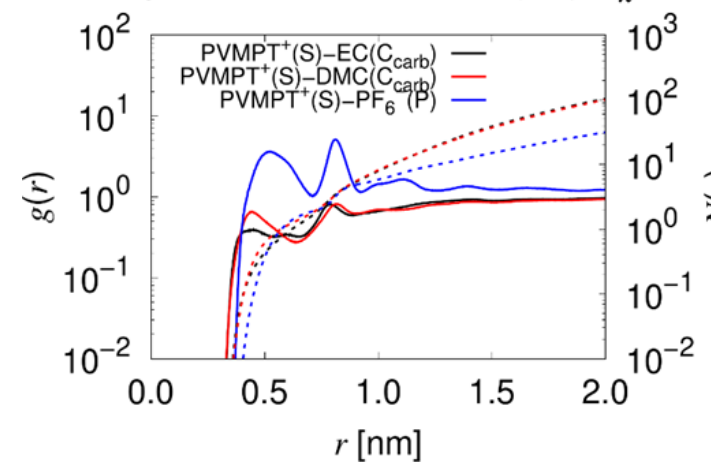

Charged PVMPT in EC:DMC (3:7), $n_{\pi}=1$

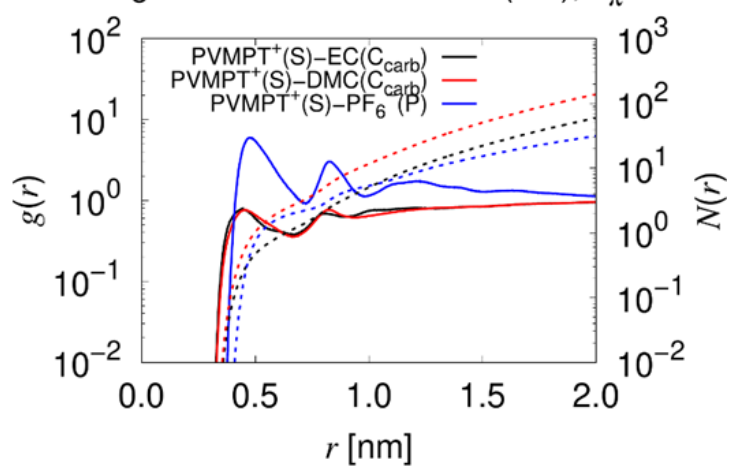

Charged PVMPT in EC:DMC (3:7), $n_{\pi}=2$

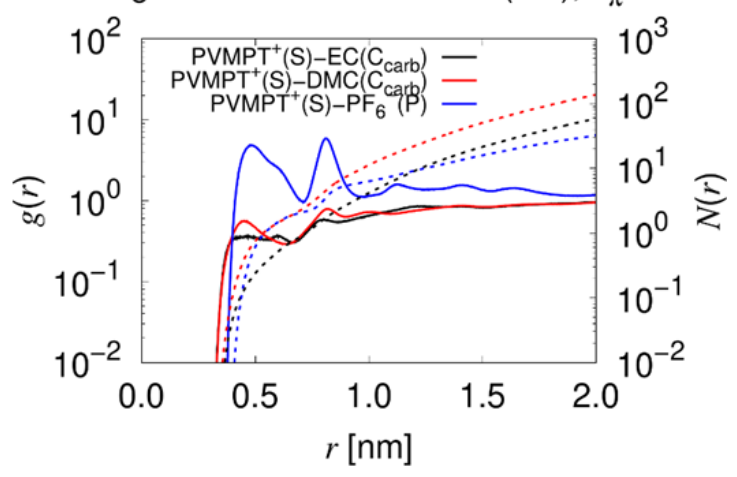

Charged PVMPT in EC:DMC (3:7), $n_{\pi}=3$

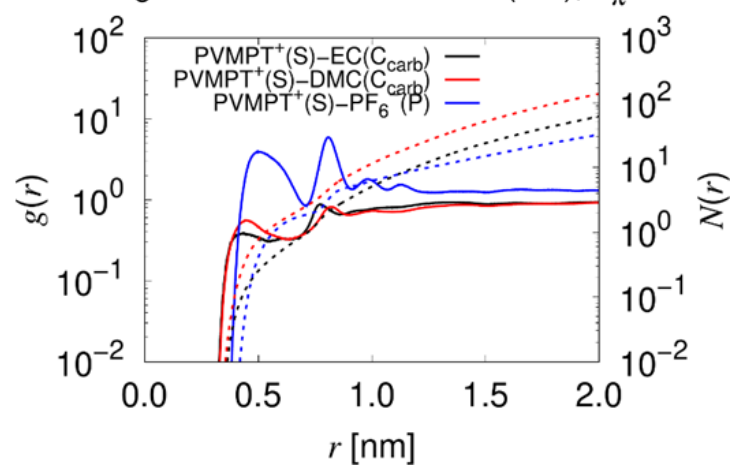

Figure S12. Radial distribution functions $g(r)$ (solid) and distance-dependent coordination numbers $N(r)$ (dashed) between the sulphur atoms of a fully oxidized PVMPT chain and the carbon atoms of the carbonate group of $E C$ and $D M C$ or the phosphorus atom of $\mathrm{PF}_{6}{ }^{-}$in $E C / D M C$ electrolytes. Both mixing ratios EC/DMC (1:1) and EC/DMC (3:7) as well as chains with monomer sequences of various length that are stabilized by $\pi$ interactions are compared. 
(a)

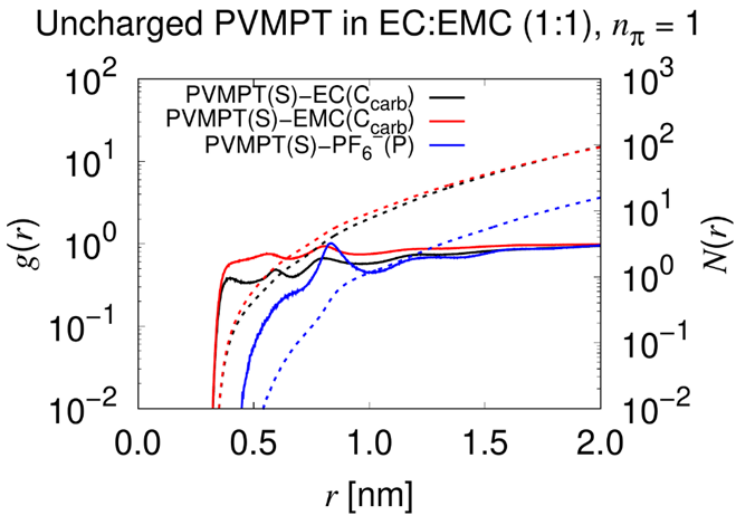

(c)

$$
\text { Uncharged PVMPT in EC:EMC, } n_{\pi}=1
$$

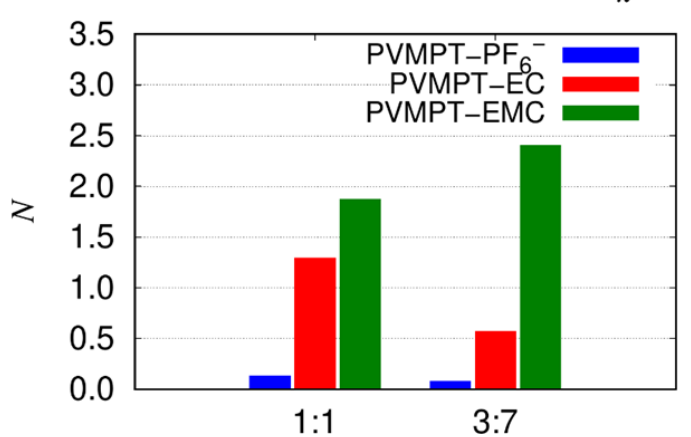

(b)

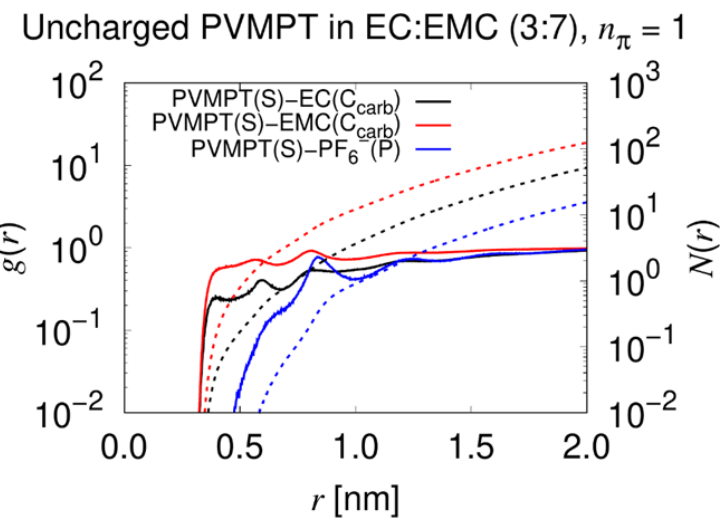

(d)

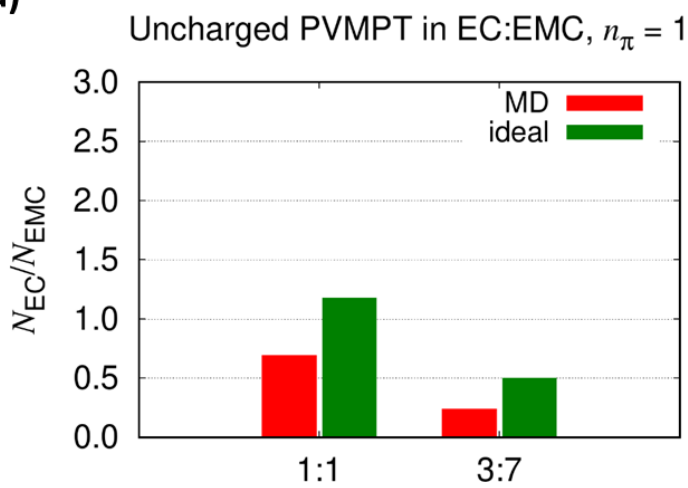

Figure S13. (a) and (b): Radial distribution functions $g(r)$ (solid) and distance-dependent coordination numbers $N(r)$ (dashed) between the sulphur atoms of the a neutral PVMPT chain and the carbon atoms of the carbonate group of EC and DMC or the phosphorus atom of $\mathrm{PF}_{6}{ }^{-}$in EC/EMC electrolytes. Both mixing ratios EC/DMC (1:1) and EC/DMC (3:7) are compared, while $\pi$-interactions are irrelevant for the neutral form of PVMPT. (c) and (d): Coordination numbers derived from (a) and (b) using the same distance criteria as in Figures S10 and S11. 


\section{References}

(1) Breneman, C. M.; Wiberg, K. B. Determining Atom - Centered Monopoles from Molecular Electrostatic Potentials. The Need for High Sampling Density in Formamide Conformational Analysis. Journal of Computational Chemistry 1989, 11, 361-373.

(2) Frisch, M. J.; Head-Gordon, M.; Pople, J. A. A Direct MP2 Gradient Method. Chemical Physics Letters 1990, 166, 275-280.

(3) Dunning, T. H. Gaussian Basis Sets for Use in Correlated Molecular Calculations. I. The Atoms Boron through Neon and Hydrogen. The Journal of Chemical Physics 1989, 90, 1007-1023.

(4) Adamo, C.; Barone, V. Toward Reliable Density Functional Methods without Adjustable Parameters The PBEO Model. The Journal of Chemical Physics 1999, 110, 6158-6170.

(5) Weigend, F.; Ahlrichs, R. Balanced Basis Sets of Split Valence, Triple Zeta Valence and Quadruple Zeta Valence Quality for $\mathrm{H}$ to Rn: Design and Assessment of Accuracy. Physical Chemistry Chemical Physics 2005, 7, 3297-3305.

(6) Grimme, S.; Ehrlich, S.; Goerigk, L. Effect of the Damping Function in Dispersion Corrected Density Functional Theory. Journal of Computational Chemistry 2011, 32, 1456-1465.

(7) Frisch, M. J.; Trucks, G. W.; Schlegel, H. B.; Scuseria, G. E.; Robb, M. A.; Cheeseman, J. R.; Scalmani, G.; Barone, V.; Petersson, G. A.; Nakatsuji, H.; Li, X.; Caricato, M.; Marenich, A. V.; Bloino, J.; Janesko, B. G.; Gomperts, R.; Mennucci, B.; Hratchian, H. P.; Ortiz, J. V.; Izmaylov, A. F.; Sonnenberg, J. L.; Williams; Ding, F.; Lipparini, F.; Egidi, F.; Goings, J.; Peng, B.; Petrone, A.; Henderson, T.; Ranasinghe, D.; Zakrzewski, V. G.; Gao, J.; Rega, N.; Zheng, G.; Liang, W.; Hada, M.; Ehara, M.; Toyota, K.; Fukuda, R.; Hasegawa, J.; Ishida, M.; Nakajima, T.; Honda, Y.; Kitao, O.; Nakai, H.; Vreven, T.; Throssell, K.; Montgomery Jr., J. A.; Peralta, J. E.; Ogliaro, F.; Bearpark, M. J.; Heyd, J. J.; Brothers, E. N.; Kudin, K. N.; Staroverov, V. N.; Keith, T. A.; Kobayashi, R.; Normand, J.; Raghavachari, K.; Rendell, A. P.; Burant, J. C.; Iyengar, S. S.; Tomasi, J.; Cossi, M.; Millam, J. M.; Klene, M.; Adamo, C.; Cammi, R.; Ochterski, J. W.; Martin, R. L.; Morokuma, K.; Farkas, O.; Foresman, J. B.; Fox, D. J., Gaussian 16 Rev. C.01 Wallingford, CT, 2016.

(8) Borges Silva, L.; Freitas, L. C. G. Structural and Thermodynamic Properties of Liquid Ethylene Carbonate and Propylene Carbonate by Monte Carlo Simulations. Journal of Molecular Structure: THEOCHEM 2007, 806, 23-34.

(9) Jorgensen, W. L.; Maxwell, D. S.; Tirado-Rives, J. Development and Testing of the OPLS All-Atom Force Field on Conformational Energetics and Properties of Organic Liquids. Journal of the American Chemical Society 1996, 118, 11225-11236.

(10) Canongia Lopes, J. N.; Deschamps, J.; Pádua, A. A. H. Modeling lonic Liquids Using a Systematic All-Atom Force Field. The Journal of Physical Chemistry B 2004, 108, 2038-2047.

(11) Canongia Lopes, J. N.; Pádua, A. A. H. Molecular Force Field for lonic Liquids Composed of Triflate or Bistriflylimide Anions. The Journal of Physical Chemistry B 2004, 108, 16893-16898.

(12) Canongia Lopes, J. N.; Pádua, A. A. H. CL\&P: A generic and Systematic Force Field for lonic Liquids Modeling. Theoretical Chemistry Accounts 2012, 131, 1129.

(13) Åqvist, J. Ion-Water Interaction Potentials derived from Free Energy Perturbation Simulations. The Journal of Physical Chemistry 1990, 94, 8021-8024.

(14) Pádua, A. A. H. Torsion Energy Profiles and Force Fields Derived from Ab Initio Calculations for Simulations of Hydrocarbon-Fluorocarbon Diblocks and Perfluoroal. The journal of Physical Chemistry A 2002, 106, 10116-10123.

(15) Neumann, M. Dipole Moment Fluctuation Formulas in Computer Simulations of Polar Systems. Molecular Physics 2006, 50, 841-858.

(16) Frenkel, D.; Smit, B. Understanding Molecular Simulation: From Algorithms to Applications; 2nd ed.; Academic Press, 2001.

(17) Hall, D. S.; Self, J.; Dahn, J. R. Dielectric Constants for Quantum Chemistry and Li-Ion Batteries: Solvent Blends of Ethylene Carbonate and Ethyl Methyl Carbonate. The Journal of Physical Chemistry C 2015, 119, 22322-22330. 
(18) Bedrov, D.; Piquemal, J.-P.; Borodin, O.; MacKerell, A. D.; Roux, B.; Schröder, C. Molecular Dynamics Simulations of Ionic Liquids and Electrolytes Using Polarizable Force Fields. Chemical Reviews 2019, 119, 7940-7995.

(19) Soetens, J.-C.; Millot, C.; Maigret, B. Molecular Dynamics Simulation of Li+BF4- in Ethylene Carbonate, Propylene Carbonate, and Dimethyl Carbonate Solvents. The Journal of Physical Chemistry A 1998, 102, 1055-1061.

(20) Borodin, O.; Smith, G. D. Quantum Chemistry and Molecular Dynamics Simulation Study of DMCEC electrolytes doped with LiPF6. The Journal of Physical Chemistry B 2009, 113, 1763-1776.

(21) Oldiges, K.; Diddens, D.; Ebrahiminia, M.; Hooper, J. B.; Cekic-Laskovic, I.; Heuer, A.; Bedrov, D.; Winter, M.; Brunklaus, G. Understanding Transport Mechanisms in lonic Liquid-Carbonate Solvent Electrolyte Blends. Physical Chemistry Chemical Physics 2018, 20, 16579-16591.

(22) Kolek, M.; Otteny, F.; Schmidt, P.; Mück-Lichtenfeld, C.; Einholz, C.; Becking, J.; Schleicher, E.; Winter, M.; Bieker, P.; Esser, B. Ultra-High Cycling Stability of Poly(vinylphenothiazine) as a Battery Cathode Material resulting from $\pi-\pi$ Interactions. Energy \& Environmental Science 2017, 10, 2271-2482. 